\title{
High Post-infection Protection after COVID-19 Among Healthcare Workers: A Population-Level Observational Study Regarding SARS-CoV-2 Reinfection, Reactivation, and Re-positivity and its Severity
}

\section{Golnar Sabetian}

Shiraz University of Medical Sciences

\section{Sepehr Shahriarirad}

Shiraz University of Medical Sciences

\section{Mohsen Moghadami}

Shiraz University of Medical Sciences

\section{Naeimehossadat Asmarian}

Shiraz University of Medical Sciences

Reza Shahriarirad ( $\nabla$ r.shahriari1995@gmail.com )

Shiraz University of Medical Sciences https://orcid.org/0000-0001-5454-495X

Mehrdad Askarian

Shiraz University of Medical Sciences

Leila Hashemizadeh Fard Haghighi

Shiraz University of Medical Sciences

Parisa Javadi

Shiraz University of Medical Sciences

\section{Research Article}

Keywords: COVID-19, PCR re-positivity, Reinfection, Relapse, Healthcare Worker

Posted Date: August 6th, 2021

DOl: https://doi.org/10.21203/rs.3.rs-772662/v1

License: (c) (i) This work is licensed under a Creative Commons Attribution 4.0 International License. Read Full License 


\section{Abstract}

Introduction: Even though over a year has passed since the coronavirus 2019 (COVID-19) outbreak, our information regarding certain aspects of the disease, such as post-infection immunity is still very limited. This study aimed to evaluate post-infection protection and COVID-19 features among healthcare workers (HCWs), during three subsequent surges.

Method: The study population consisted of all HCWs in either public or private hospitals in Fars province, Southern Iran from 20 April 2020 up to $20^{\text {th }}$ February 2021. We calculated the rate of infection as the number of individuals with positive PCR tests divided by the cumulative number of person-days at risk. Poisson regression was utilized to calculate the adjusted rate ratio and estimated protection.

Results: During the study period, a total of 30,546 PCR tests were performed among HCWs, of which 13,749 HCWs were positive. Among a total of 141 diagnosed cases who experienced a second episode of COVID-19, 44 (31.2\%) cases of reactivation and relapse, and 97 (68.8\% of infected and $1.81 \%$ of total HCWs) cases of reinfection was observed. The daily rate of infection was 4.72 for previously infected $\mathrm{HCWs}$, while 2.20 for HCWs without previous infection. The estimated protection against repeat infection after a previous SARS-CoV-2 infection was $94.8 \%$ (95\% Cl: 93.6-95.7).

Conclusion: Re-positivity, relapse, and reinfection of SARS-CoV-2 are quite rare in the population of HCWs. Also, after a first episode of infection, estimated protection of $94.8 \%$ was achieved against repeat infections.

\section{Introduction}

Since the beginning of the COVID-19 pandemic, according to WHO, SARS-CoV-2 has infected over 165 million people and over 3.4 million instances of death have occurred all over the world (1). While the absence of a pre-existing immunity prior to SARS-CoV-2 exposure plays a key role in the rapid spread of COVID-19, the duration and degree of protection acquired after an infection of this kind, or after vaccination, is an equally important concept. Understanding the extent of post-infection immunity can provide useful information for the implication of appropriate intervention strategies.

When investigating post-infection immunity, certain considerations are of key-value; identification of measurable surrogate markers and correlates of protection along with providing a proper definition for endpoints, i.e. onward transmission, disease prevention, or death, are among such considerations which arrange for a more precise investigation $(2,3)$.

Currently, evidence suggesting SARS-CoV-2 post-infection immunity is on the rise. While some single and small case studies have reported instances of COVID-19 reinfection (4-7), two large studies in the UK have found that protective immunity could last for approximately $5 \rrbracket 6$ months $(2,8)$. This suggests that most people are provided with some immunity after the first infection, and to acquire secondary and repeat infections is considered to be quite rare. 
Based on available information and the undeniable concern regarding the possibility of a recurrence of COVID-19, this study was designed to assess reinfection, relapse, and re-positivity of SARS-CoV-2 in HCWs along with comparing manifested clinical symptoms during each episode of a patient's infection.

\section{Material And Method}

\section{Study design and Surveillance system}

In this cross-sectional population-level observational study, individual-level data regarding infected cases among HCWs and hospital staff working in Fars province, Southern Iran, were obtained from 20th April to 18 February 2021 (305 days). During this period, Fars province experienced three surges of COVID-19 cases, including 1th of June 2020-15th August 2020, 28th September 2020-12th December 2020, and 21st March 2021-18th February 2021 (end of study period and date of data collection).

The study population consisted of all healthcare personnel working in either public or private hospitals in Fars province. These data were recorded from 44 private and community hospitals throughout the province. Surveillance systems and testing were done for individuals based on the presentation of symptoms or in cases with unprotected close contact with confirmed COVID-19 cases, as previously described $(9,10)$. RT-PCR assays were performed according to the protocol established by the WHO and previous studies (11-13). Deidentified data were obtained from the database, which has a generic research ethics committee, Health Research Authority, and Confidentiality Advisory Group approvals.

\section{Data collection}

The patient's demographic information, signs and symptoms, radiological findings, occupation, and working location, were recorded by contacting the patient and filling out a pre-designed form. Also, signs and symptoms after recovery were documented. Furthermore, data regarding the total number of HCWs performed tests, and also the total number of infected cases in the mentioned timeline in the studied centers along with features such as age, sex, and occupation were recorded for comparison.

Also, the patients computed tomography (CT) scans were deidentified and evaluated individually by a specialist who was blinded to the patient's status and previous/further CT scans.

\section{Definition of infections, re-positivity, relapse, and reinfections}

A COVID-19 episode was defined by (i) at least one recent major clinical sign of COVID-19 including fever or chills, febrile flu-like syndrome, dyspnea, anosmia, or dysgeusia; and (ii) a positive SARS-CoV-2 RT-PCR test. Patients were not included if a differential diagnosis (amongst which bacterial, fungal, or other viral superinfection, thrombo-embolic complication, secondary organizing pneumonia, or interstitial lung disease) could explain the symptom recurrence. 
The definition of reinfection, relapse, and re-positivity of COVID-19 was assigned based on previous reports. $(14,15)$

- Re-positivity, according to the Centers for Disease Control and Prevention (CDC), is described as a positive PCR for COVID-19 during a 90-day time frame probably represents prolonged shedding rather than reinfection. (15) SARS-CoV-2 RT-PCR re-positivity describes positive RT-PCR following negative tests in an asymptomatic patient up to 90 days from the first episode. These cases probably do not represent replicative viruses and do not necessitate isolation. Low viral load is usually a feature of re-positivity.

- COVID-19 relapse (also described in the literature as 'recrudescence' or 'recurrence' or 'reactivation') clinical recurrence of symptoms compatible with COVID-19 accompanied by positive/ persisting RTPCR within 90 days of primary infection, and supported by the absence of epidemiological exposure or another cause of the illness. (14)

- Yahav et al (14) defined reinfection with COVID-19 in the context of clinical practice as clinical recurrence of symptoms compatible with COVID-19, accompanied by positive PCR test $(\mathrm{Ct}<35)$, more than 90 days after the onset of the primary infection, supported by close-contact exposure or outbreak settings, and no evidence of another cause of infection. In the presence of epidemiological risk factors (i.e., significant exposure), reinfection should be considered during the first 90 days, if clinical symptoms of the first episode resolved and two PCR tests were negative before the new episode. For epidemiological purposes, reinfection could be defined as any positive RT-PCR test (Ct values $<35$ ) more than 90 days from the first episode, regardless of symptoms. Since confirmation by genotypic assays is time and resource-consuming, any case of suspected reinfection should be considered for isolation.

We classified health care workers according to their baseline RT-PCR status. Those with only negative RTPCR for SARS-CoV-2 were considered to be at risk for infection from their first test until either the end of the study or their first PCR-positive test, whichever occurred earlier. Those with a positive PCR were considered to be at risk for infection (or reinfection) from 90 days after their first positive antibody result to either the end of the study or their next PCR-positive test, whichever occurred earlier, irrespective of subsequent seroreversion (i.e., any negative PCR tests occurring later). Patients with a positive PCR during the initial 90 days of our study were considered previously infected unless succeeding to a negative PCR. Regarding the days at risk, exposed periods are periods of follow-up time contributed by individuals with the previous infection while unexposed periods are contributed by individuals without previous infection. In cases where the status of an individual changed from uninfected to infected, the patient remained in the follow-up while contributing to the previously infected group. The adapted method was based on a study by population-based study by Hansen et al. (16)

For the purpose of evaluating reinfection, we included all HCWs with a diagnosis of COVID-19 either based on positive PCR or clinical assessment during our study period. The date of first COVID-19 diagnosis in each individual was recorded along with their follow-up and further PCR or clinical evaluations. Each individual with a diagnosis of COVID-19, either based on PCR or clinical assessment, 
was followed up from the time of their first test, irrespective of the date and whether they had a positive or negative result, until the end of our study period. The study and analytic design and cut-off of 90 days was assigned based on previous reports and evidence in this regard, (14-16) We subcategorized possible reinfections by symptom status to highlight those with stronger evidence and provide comparability with definitions used elsewhere

\section{Statistical analysis}

We calculated the rate of infection as the number of individuals with positive PCR tests divided by the cumulative number of person-days at risk. We calculated the number of days at risk for each individual in the sample as the number of days from three months after the first positive test, until the first positive test, or the end of our study period, whichever came first. We censored follow-up time in the event of death. This non-informative censoring mechanism essentially assumed a similar infection rate would have been observed among those who died if they had survived, as was observed among the survivors with the same exposure status (whether previously infected or uninfected). We calculated the adjusted rate ratio (RR) and accompanying $95 \% \mathrm{Cl}$ using Poisson regression. Protection against repeat infections was calculated as 1 - adjusted RR, analogous to the method of estimating vaccine effectiveness from observational data. We used Poisson regression to model the incidence of PCR-positive infection per atrisk day according to baseline antibody status, adjusting for incidence over time. We report proportions calculated using exact (Clopper-Pearson) 95\% Cls. We did all analyses and graph generation using SPSS version 22.0 and Excel software.

\section{Results}

The capacity for COVID-19 screening and performing PCR testing for SARS-CoV-2 in Iran increased rapidly over 2020, from the first tests on 20 April 2020 up to 20th February 2021, when approximately a daily average of 45.22 tests among HCWs and 1,947 tests among the general population was performed. By the end of our study period, $13,749 \mathrm{HCWs}$-more than two-thirds of the population of 22,195 active HCWs in the province-had been tested at least once. Also, a total of 30,546 PCR tests were performed during the study period. Figure 1 demonstrates the frequency of positive COVID-19 among HCWs compared to the total test performed among healthcare workers, while Fig. 2 illustrates the related proportion.

There were no cases of mortality in our study. Patients who were diagnosed with COVID-19 remained in follow-up. Based on the database, 5349 (38.9\%) HCWs contracted the COVID-19 and 8400 (61.1\%) remained uninfected by the virus. Among the infected patients, $943(17.6 \%)$ had a positive PCR test during the initial 90 days of the study (and therefore contributed to the previously infected group). Figure 3 demonstrates the frequency of positive COVID-19 among the general population and healthcare workers, while Fig. 4 demonstrates the related proportion.

Among the patients in our follow-up, 141 (2.64\%) had a second episode of COVID-19 diagnosis after the complete alleviation of symptoms and/or a negative PCR of SARS-CoV-2 following the treatment of the 
first episode. The interval between diagnosis of COVID-19 was categorized as 90 and above days, and before 90 days and assigned as reinfection and reactivation, respectively. Based on our data, among a total of 141 diagnosed cases of COVID-19, a total of 44 (31.2\%) cases of reactivation and relapse, and 97 ( $68.8 \%$ of infected and $1.81 \%$ of total HCWs) cases of reinfection was observed. Table 1 demonstrates the overall features of the HCWs with COVID-19, while Table 2 provides a comparison of the first and second episodes of COVID-19 in these patients. 
Table 1

Overall features of the healthcare workers with coronavirus disease (COVID-19)

\begin{tabular}{|c|c|c|c|c|c|c|}
\hline Variables & & Total & Reinfection & Relapse & $\begin{array}{l}\mathrm{Re}- \\
\text { positivity }\end{array}$ & $\begin{array}{l}\text { P- } \\
\text { value* }\end{array}$ \\
\hline Age; mean \pm SD & & $\begin{array}{l}35.00 \pm \\
7.18\end{array}$ & $\begin{array}{l}35.70 \pm \\
7.43\end{array}$ & $\begin{array}{l}33.46 \pm \\
7.95\end{array}$ & $\begin{array}{l}33.43 \pm \\
5.75\end{array}$ & 0.285 \\
\hline \multirow[t]{4}{*}{ Age group; n (\%) } & $21-30$ & $49(35)$ & $31(32.6)$ & $6(40)$ & $12(40)$ & \multirow[t]{4}{*}{0.791} \\
\hline & $31-40$ & $64(45.7)$ & $44(46.3)$ & $7(46.7)$ & 13(43.3) & \\
\hline & $41-50$ & $23(16.4)$ & $17(17.9)$ & $1(6.7)$ & $5(16.7)$ & \\
\hline & $51-60$ & $4(2.9)$ & $3(3.1)$ & $1(6.7)$ & $0(0)$ & \\
\hline \multirow[t]{2}{*}{ Gender, n (\%) } & Male & 53 (37.6) & $36(37.9)$ & $6(37.5)$ & $11(36.7)$ & \multirow[t]{2}{*}{0.993} \\
\hline & Female & $88(62.4)$ & $59(62.1)$ & $\begin{array}{l}10 \\
(62.5)\end{array}$ & $19(63.3)$ & \\
\hline \multirow[t]{4}{*}{ Occupation; n (\%) } & Specialist \&GP & $8(5.6)$ & $5(5.2)$ & $1(5.9)$ & $2(6.9)$ & \multirow[t]{4}{*}{0.853} \\
\hline & nurse & $76(53.1)$ & $48(49.5)$ & $\begin{array}{l}10 \\
(58.8)\end{array}$ & $18(62.1)$ & \\
\hline & technician & $16(11.2)$ & $12(12.4)$ & $1(5.9)$ & $3(10.3)$ & \\
\hline & office & $43(30.1)$ & 32 (33.0) & $5(29.4)$ & $6(20.7)$ & \\
\hline \multirow[t]{2}{*}{ Hospital; n (\%) } & $\begin{array}{l}\text { COVID-19 } \\
\text { Specific }\end{array}$ & 46 (31.9) & $24(24.7)$ & $7(41.2)$ & $15(50)$ & \multirow[t]{2}{*}{0.024} \\
\hline & $\begin{array}{l}\text { Non-COVID-19 } \\
\text { hospital }\end{array}$ & $98(68.1)$ & 73 (75.3) & $\begin{array}{l}10 \\
(58.8)\end{array}$ & $15(50)$ & \\
\hline \multirow{2}{*}{$\begin{array}{l}\text { Comorbid } \\
\text { diseases; n (\%) }\end{array}$} & yes & $25(17.4)$ & 17 (17.5) & $3(17.6)$ & $5(16.7)$ & \multirow[t]{2}{*}{0.994} \\
\hline & no & $\begin{array}{l}119 \\
(82.6)\end{array}$ & $80(82.5)$ & $\begin{array}{l}14 \\
(82.4)\end{array}$ & 25 (83.3) & \\
\hline \multicolumn{7}{|c|}{$\begin{array}{l}\text { Re-positivity: positive RT-PCR following negative tests in an asymptomatic patient up to } 90 \text { days from } \\
\text { the first episode; Relapse: clinical recurrence of symptoms compatible with COVID-19 accompanied } \\
\text { by positive/ persisting RT-PCR within } 90 \text { days of primary infection, and supported by the absence of } \\
\text { epidemiological exposure or another cause of the illness; Reinfection: }>90 \text { days or }<90 \text { days + if } \\
\text { clinical symptoms of the first episode resolved and two PCR tests were negative before the new } \\
\text { episode. }\end{array}$} \\
\hline \multicolumn{7}{|c|}{ * Chi-square/Fisher's exact test } \\
\hline
\end{tabular}


Table 2

Comparison of first and second episode of COVID-19 among healthcare workers

\begin{tabular}{|c|c|c|c|c|}
\hline \multirow{2}{*}{$\begin{array}{l}\text { Variable } \\
\text { Reinfection; } n=97\end{array}$} & \multicolumn{4}{|c|}{ Frequency (\%) } \\
\hline & Total & First episode & Second episode & Overlap \\
\hline \multicolumn{5}{|l|}{ Symptoms } \\
\hline Gastrointestinal & $56(57.7)$ & $29(29.9)$ & $49(50.5)$ & $22(22.7)$ \\
\hline Neurological & $81(83.5)$ & $46(47.4)$ & $75(77.3)$ & $40(41.2)$ \\
\hline Respiratory & 75 (77.3) & $42(43.3)$ & $71(73.2)$ & $38(39.2)$ \\
\hline Asymptomatic & $23(23.7)$ & $22(22.7)$ & $1(1)$ & $0(0)$ \\
\hline Flu like symptom & $96(99)$ & $65(67)$ & 95 (97.9) & $64(66)$ \\
\hline Cardiovascular & $50(51.5)$ & $19(19.6)$ & $47(48.5)$ & $16(16.5)$ \\
\hline Others & $14(14.4)$ & $8(8.2)$ & $8(8.2)$ & $2(2.1)$ \\
\hline CT \& CXR involvement & $49(50.5)$ & $26(27)$ & $38(39)$ & $15(14)$ \\
\hline Hospitalization & 14 (14.4) & $8(8)$ & $7(7)$ & $1(1)$ \\
\hline ICU Admission & $3(3.1)$ & $0(0)$ & $3(3.1)$ & $0(0)$ \\
\hline O2 therapy & $2(2)$ & $2(2)$ & $0(0)$ & $0(0)$ \\
\hline \multicolumn{5}{|l|}{ Relapse; $n=15$} \\
\hline \multicolumn{5}{|l|}{ Symptoms } \\
\hline Gastrointestinal & $13(87)$ & $5(29.4)$ & $12(70.6)$ & $4(23.5)$ \\
\hline Neurological & $15(100)$ & $11(64.7)$ & $14(82.4)$ & $10(58.8)$ \\
\hline Respiratory & $13(87)$ & $6(35.3)$ & $12(70.6)$ & $5(29.4)$ \\
\hline Asymptomatic & $4(23.5)$ & $4(23.5)$ & $1(5.9)$ & $1(5.9)$ \\
\hline Flu like symptom & $14(93.3)$ & $12(70.6)$ & $14(93.3)$ & $12(70.6)$ \\
\hline Cardiovascular & $9(6)$ & $4(23.5)$ & $8(47.1)$ & $3(17.6)$ \\
\hline Others & $2(13.3)$ & $1(5.9)$ & $1(5.9)$ & $0(0)$ \\
\hline CT \& CXR involvement & $12(8)$ & $8(53)$ & $9(60)$ & $5(33)$ \\
\hline Hospitalization & $2(13.3)$ & $1(6.7)$ & $2(13.3)$ & $1(6.7)$ \\
\hline ICU Admission & $0(0)$ & $0(0)$ & $0(0)$ & $0(0)$ \\
\hline O2 therapy & $0(0)$ & $0(0)$ & $0(0)$ & $0(0)$ \\
\hline
\end{tabular}




\begin{tabular}{|lllll|}
\hline Variable & \multicolumn{3}{l|}{ Frequency (\%) } \\
\hline Symptoms & & & \\
\hline Gastrointestinal & $17(58.6)$ & $8(26.7)$ & $14(46.7)$ & $5(16.7)$ \\
\hline Neurological & $23(79)$ & $13(43.3)$ & $20(66.7)$ & $10(33.3)$ \\
\hline Respiratory & $22(76)$ & $7(23.3)$ & $19(63.3)$ & $4(13.3)$ \\
\hline Asymptomatic & $0(0)$ & $0(0)$ & $0(0)$ & $0(0)$ \\
\hline Flu like symptom & $29(100)$ & $24(80)$ & $27(90)$ & $22(75.8)$ \\
\hline Cardiovascular & $14(48.2)$ & $4(13.3)$ & $14(46.7)$ & $4(13.3)$ \\
\hline Others & $3(10.3)$ & $2(6.7)$ & $1(3.3)$ & $0(0)$ \\
\hline CT \& CXR involvement & $16(55.2)$ & $5(17)$ & $14(48)$ & $3(10)$ \\
\hline Hospitalization & $6(20.7)$ & $1(3.4)$ & $6(20.7)$ & $1(3.4)$ \\
\hline ICU Admission & $0(0)$ & $0(0)$ & $0(0)$ & $0(0)$ \\
\hline O2 therapy & $0(0)$ & $0(0)$ & $0(0)$ & $0(0)$ \\
\hline
\end{tabular}

For the purpose of analysis, repositivity and relapse were categorized into one group and compared with reinfection based on the patients' clinical features. There was no significant difference among the symptoms of patients with COVID-19 reinfection compared with HCWs with Relapse/Repositivity ( $\mathrm{P}=$ 0.650). Also, there was no significant difference among the symptoms in the first episode, second episode, and overlapping between the two episodes among the reinfection and Relapse/Repositivity group ( $P=0.442,0.054$, and 0.162 , respectively). There was no significant difference among the total amount of radiological involvement, occurrence during the first episode, second episode, and overlapping of the two episodes among the reinfection and Relapse/Repositivity group $(P=0.440,0.801,0.369$, and 0.733 respectively). There was also no significant difference among the total need for hospitalization, frequency of hospitalization during the first and second episode, and overlapping of hospitalization among the reinfection and Relapse/Repositivity group $(P=0.120,0.458,0.085$, and 0.194 respectively). There was also no significant difference in the need for ICU admission among the two groups $(P=0.247)$. As established, there was no significant difference among the clinical presentation of HCWs with reinfection compared to the relapse/repositivity group.

For the assessment of infection rates and relative protection, the participants were followed throughout the study period of 304 days, with 5349 (38.90\%) individuals contributing exposed time periods and 12,806 (93.14\%) contributing unexposed time periods, while 4406 contributing to both unexposed and exposed time periods, with a total of 97 reinfections. Among the 12806, 4406 became infected during the study, contributing 41571 days, while 8400 remained uninfected, contributing 1,965,106 days, throughout the study period. The daily rate of infection was 4.72 for previously infected HCWs, while 2.20 for HCWs without previous infection. The adjusted RR of infection was $0.052(95 \% \mathrm{Cl}$ : $0.043-0.064)$ among those 
who previously tested positive compared with those who had previously only tested negative. The estimated protection against repeat infection after a previous SARS-CoV-2 infection was $94.8 \%$ (95\% Cl: 93.6-95.7) (Table 3).

Table 3

Comparison of infection and reinfection rates and estimated protection against reinfection with SARSCoV-2in healthcare workers based on days of exposure and during follow-ups

\begin{tabular}{|c|c|c|c|c|c|c|}
\hline Period & Population & $\begin{array}{l}\text { Confirmed } \\
\text { New } \\
\text { infection } \\
\text { during } \\
\text { follow-up }\end{array}$ & $\begin{array}{l}\text { Person } \\
\text { days of } \\
\text { follow-up }\end{array}$ & $\begin{array}{l}\text { Infection rate } \\
\text { during follow- } \\
\text { up (Per } 1000 \\
\text { population) }\end{array}$ & $\begin{array}{l}\text { Adjusted } \\
\text { Rate } \\
\text { ratio } \\
(95 \% \mathrm{Cl})\end{array}$ & $\begin{array}{l}\text { Estimated } \\
\text { Protection } \\
(95 \% \mathrm{Cl})\end{array}$ \\
\hline Exposed & 5349 & 97 & 20562 & 4.72 & $\begin{array}{l}0.052 \\
(0.043- \\
0.064)\end{array}$ & $\begin{array}{l}94.8 \% \\
(93.6- \\
95.7)\end{array}$ \\
\hline Unexposed & $12806^{\mathrm{a}}$ & 4406 & $2,006,677^{b}$ & 2.20 & 1 (ref) & - \\
\hline \multicolumn{7}{|c|}{ 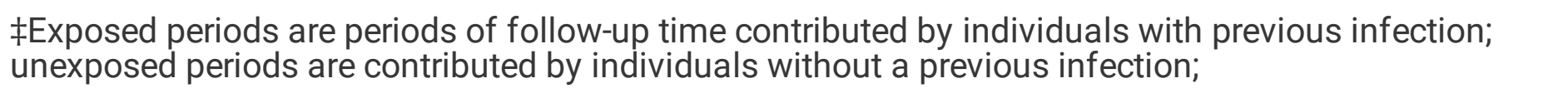 } \\
\hline \multicolumn{7}{|c|}{ Infection rate during follow-up is calculated based on confirmed new / person days/1000; } \\
\hline \multicolumn{7}{|c|}{ a: 4406 infected during study and 8400 uninfected } \\
\hline b: 41571 rel & ed to infecte & group and 1 , & 106 related & uninfected groı & & \\
\hline
\end{tabular}

We aimed to evaluate the infection rates during and in-between surges among HCWs and the general population. (Fig. 5). During the first surge (ie, before June 2020), 1115 people were PCR positive, among eligible PCR-positive individuals 75 (6.72\%) Healthcare workers were PCR positive, after the first surge (ie, before July 2020) 2,301 people were positive, among eligible PCR-positive individuals 33 (1.43\%) Healthcare worker were PCR positive. During the second surge (ie, before Auguste, 2020), 32,001 people were PCR positive, of whom 1075 (3.35\%) Healthcare workers had tested positive and after the second surge (ie, before October 2020) 14,608 people were positive, among eligible PCR-positive individuals 461 (3.15\%) Healthcare worker were PCR positive. During the third surge (ie, before December 2020), 102,294 people were PCR positive, of whom 3,370 (3.29\%) Healthcare workers had tested positive and after the third surge (ie, before Murch, 2021) 14,897 people were positive, among eligible PCR-positive individuals 335 (1.92\%) Healthcare worker were PCR positive. There was a significant difference among HCW to general population proportion between the first surge and the second surge $(P<0.001)$ and also between the first surge and the third surge $(P<0.001)$, but not between the second surge and the third surge $(P=$ $0.600)$

It is also worth mentioning that $5855 \mathrm{HCWs}$ underwent one PCR, 470 two times, 42 three times, and three underwent four episodes of PCR.

\section{Discussion}


In this study, we evaluated different episodes of COVID-19 in HCWs using terms provided by recent reports (17), i.e. re-positivity, relapse, and reinfection, as accommodating investigative measures. Following the main objective of our study, we found that after an episode of SARS-CoV-2 infection, HCWs have an estimated protection of $94.8 \%$ against repeated infections. The finding of this study provides useful insight into the events that occur after each COVID-19 episode, and they may also affect how we consider our approaches to providing proper and ideal protective measures.

Since the beginning of the pandemic, HCWs have been at a very high risk of SARS-CoV-2 infection. Ever since numerous reports have illustrated this threat on various occasions. A study on Australian HCWs identified the risk of SARS-CoV-2 infection to be three folds higher in HCWs than in the general population (18). While our study shows that during the first to third surges of COVID-19 occurring from 20th Apr 2020 to 18th Feb 2021 in Fars province, Iran, there were 1115, 32,001, and 102,294 PCR-positive individuals of the general population, $75(6.72 \%), 1075$ (3.35\%), and 3370 (3.29\%) of this population was comprised of HCWs, respectively. Laursen et al. similarly reported positive PCR tests in $4.9 \%$ of Danish and Swedish HCWs from June to Aug 2020, while also suggesting customer/patient contact to be a prominent factor contributing to seropositivity (19). We also reported in a previous study an infection rate of $5.62 \%$ among HCWs in our province, however, this report was during the early periods of the pandemic in which proper efficient measures of protection were not met. (9) As our data and figures demonstrate, infection rates decreased from one surge to the next, while also the sensitivity of PCR performance increased and the proportion verged towards $100 \%$. This demonstrated the increased capability in the precise diagnosis of COVID-19 and appropriate screening measures applied in healthcare centers.

The risk of SARS-CoV-2 infection and/or reinfection is considered to be higher in HCWs than in the general population. While a number of seroprevalence studies have similarly commented on this matter (20-23), high occupational exposure and ease of access to testing equipment (PCR, CT) are thought to be the two main reasons responsible for the discrepancy between this group and the general population. An Australian simulation study suggested that 6 to 14 staff members contact an infected patient in the emergency room every day (24). Also, Iversen et al. found that front-line HCWs are at 1.3 times higher risk of infection compared to other HCWs (25). Furthermore, due to higher knowledge about COVID-19 and its symptoms, and also easier access to testing equipment, more tests are performed by HCWs, deeming it less likely for an infection to go unreported. On this subject, a study conducted by Hansen et al. which was based on Denmark's national database found that each HCW had a median of 10 PCR tests (IQR 912) performed in the year 2020 (26). However, as demonstrated in our results, the proportion of positive tests to total performed tests While HCWs have higher knowledge of COVID-19 and its symptoms, a study by Erfani et al. showed that despite having this awareness, this group display lower levels of positive practice towards COVID-19 (27). This can also be a matter of concern in case of reinfection where a pseudo sense of safety after prior infection might cause HCWs to underestimate their need for protective measures.

As previously mentioned, the main finding of our study suggests that after an episode of SARS-CoV-2 infection, HCWs gain estimated protection of $94.8 \%$ against subsequent infections of this type. The 
results of our study endorse findings of several other studies conducted in the US, the UK, Denmark, and Qatar which report reinfections to be rare, occurring in less than $1 \%$ of all COVID-19 cases $(2,8,26,28$, 29). In the Denmark study, 28875 individuals contributed to exposed time periods, 138 of which were later diagnosed with reinfections. This study reported estimated protection of $78.8 \%$ among the participants (26). An interesting finding of the UK study of $20000 \mathrm{HCWs}$ was that after 5 months, the risk of SARSCoV-2 infection was reduced by $83 \%$ (8). A reason for the high estimated rates of protection in HCWs against repeat infections might be due to them being at a higher risk of primary infection compared to the general population, leading to higher levels of acquired post-infection immunity lasting up to 6 months (2, 8), and therefore, deeming them less likely to be prone to reinfections. Nevertheless, proper investigation is required to further validate this statement.

In the definition of reinfection, serology could be either positive or negative, thus, it does not provide any value. Positive serology is indicative of either insufficient antibody titers for eliminating viral agents or a possibly different strain of virus compared to the previous infection which is not recognized by the antibodies. On the other hand, a negative serology suggests a lack of adequate immune response or antibody waning (17). Nevertheless, even a non-sterilizing immune response can produce some level of protection against severe disease. This can be seen in an event of reinfection described by To et al., which was later explained to be milder than usual reinfections due to priming of the adaptive immune response during a previous episode of SARS-CoV-2 (6).

Some studies pose a different view on this matter suggesting that reinfections may occur at a more severe level due to antibody-dependent enhancements, or the appearance of other strains of the virus with higher virulence $(7,30)$. A more severe reinfection event may occur as the result of antibodydependent enhancement, or it may depend upon the virulence of the strain and its inoculum $(7,30)$ Also, observations of more severe cases may also reflect a selection bias towards testing symptomatic cases (31)

Among the limitations of our study, there is recall bias, where the participants may or may not be able to recollect the specifics of their previous infections. Another is that details regarding infection among the general population were not available to compare demographic features of HCWs to the general population. Hansed et al reported little evidence that the degree of protection against repeat infection as measured by PCR positivity conferred by the previous infection varied by age group below age 65 years. However, protection against repeat infection among those aged 65 years and older was lower than among younger age groups. (16) Further larger-scale studies in this regard are warranted.

\section{Conclusion}

Re-positivity, relapse, and reinfection of SARS-CoV-2 are quite rare in the population of HCWs. Also, after the first episode of infection, estimated protection of $94.8 \%$ was achieved against repeat infections.

\section{Abbreviations}


Cl: Confidence interval; COVID-19: Coronavirus disease 2019;CXR: Chest x-ray; CT: Computerized tomography; HCW: Healthcare worker; PCR: Polymerase chain reaction; SARS-CoV-2: severe acute respiratory syndrome coronavirus 2; SD: Standard deviation

\section{Declarations}

\section{Ethical approval of the study}

Written informed consent was obtained from the patients in our study. The purpose of this research was completely explained to the patients and they were assured that their information will be kept confidential by the researcher. The present study was approved by the Medical Ethics Committee of the academy (Ethical code: IR.SUMS.REC.1399.034).

\section{Consent for publication}

Written consent was obtained from the patients regarding the publication of this report.

\section{Availability of data and materials}

SPSS data of the participant can be requested from the authors. Please write to the corresponding author if you are interested in such data.

\section{Competing interests}

The authors declare that they have no competing interests.

\section{Funding}

No financial support was received for this study.

\section{Authors contribution}

R.S: Investigation, Conceptualization, Methodology, Validation, Formal analysisWriting - Original Draft, Review \& Editing; G.S: Investigation, Conceptualization, Supervision, Review \& Editing; S.S: Writing Original Draft; M.M: Supervision;N.A: Formal analysis; M.A: Supervision, Methodology;L.H: Resources, Data Curation;P.J: Resources, Data Curation,

\section{Acknowledgments}

We would like to thank all the medical staff and healthcare workers for their help during data collection. We express our gratitude to the Statistics \& GIS office of Shiraz University of Medical Sciencesand its members (managed by Mohammad Habibi, MD;Masoumeh Khosravi, MSc.Statistics; and Mohammadreza Serati/MSc.IT) for providing us with the necessary supplementary data to complete our study, and also all the healthcare workers for their assistance in filling out our questionnaire. 


\section{References}

1. WHO Coronavirus (COVID-19) Dashboard [Available from: https://covid19.who.int/.

2. Lumley SF, O'Donnell D, Stoesser NE, Matthews PC, Howarth A, Hatch SB, et al. Antibody status and incidence of SARS-CoV-2 infection in health care workers. New England Journal of Medicine. 2021;384(6):533-40.

3. Plotkin SA, Plotkin SA. Correlates of vaccine-induced immunity. Clinical infectious diseases. 2008;47(3):401-9.

4. Gupta V, Bhoyar RC, Jain A, Srivastava S, Upadhayay R, Imran M, et al. Asymptomatic reinfection in 2 healthcare workers from India with genetically distinct severe acute respiratory syndrome Coronavirus 2. Clinical Infectious Diseases. 2020.

5. Lee J-S, Kim SY, Kim TS, Hong KH, Ryoo N-H, Lee J, et al. Evidence of severe acute respiratory syndrome coronavirus 2 reinfection after recovery from mild coronavirus disease 2019. Clinical Infectious Diseases. 2020.

6. To KK-W, Hung IF-N, Ip JD, Chu AW-H, Chan W-M, Tam AR, et al. COVID-19 re-infection by a phylogenetically distinct SARS-coronavirus-2 strain confirmed by whole genome sequencing. Clinical infectious diseases. 2020.

7. Tillett RL, Sevinsky JR, Hartley PD, Kerwin H, Crawford N, Gorzalski A, et al. Genomic evidence for reinfection with SARS-CoV-2: a case study. The Lancet Infectious Diseases. 2021;21(1):52-8.

8. Hall VJ, Foulkes S, Charlett A, Atti A, Monk EJ, Simmons R, et al. Do antibody positive healthcare workers have lower SARS-CoV-2 infection rates than antibody negative healthcare workers? Large multi-centre prospective cohort study (the SIREN study), England: June to November 2020. medRxiv. 2020:2021.01. 13.21249642.

9. Sabetian G, Moghadami M, Haghighi LHF, Fallahi MJ, Shahriarirad R, Asmarian N, et al. COVID-19 infection among healthcare workers: a cross-sectional study in southwest Iran. 2020.

10. Shahriarirad R, Khodamoradi Z, Erfani A, Hosseinpour H, Ranjbar K, Emami Y, et al. Epidemiological and clinical features of 2019 novel coronavirus diseases (COVID-19) in the South of Iran. BMC infectious diseases. 2020;20(1):1-12.

11. Organization WH. Laboratory testing for coronavirus disease 2019 (COVID-19) in suspected human cases: interim guidance, 2 March 2020. World Health Organization; 2020.

12. Organization WH. Coronavirus disease (COVID-19) technical guidance: Laboratory testing for 2019nCoV in humans 2020 [

13. Corman VM, Landt O, Kaiser M, Molenkamp R, Meijer A, Chu DK, et al. Detection of 2019 novel coronavirus (2019-nCoV) by real-time RT-PCR. Eurosurveillance. 2020;25(3).

14. Yahav D, Yelin D, Eckerle I, Eberhardt CS, Wang J, Cao B, et al. Definitions for coronavirus disease 2019 reinfection, relapse and PCR re-positivity. Clinical Microbiology and Infection. 2021;27(3):315-8.

15. Control CfD, Prevention. Duration of isolation and precautions for adults with COVID-19. 2020. 2020. 
16. Hansen CH, Michlmayr D, Gubbels SM, Mølbak K, Ethelberg S. Assessment of protection against reinfection with SARS-CoV-2 among 4 million PCR-tested individuals in Denmark in 2020: a population-level observational study. The Lancet. 2021.

17. Yahav D, Yelin D, Eckerle I, Eberhardt CS, Wang J, Cao B, et al. Definitions for COVID-19 reinfection, relapse and PCR re-positivity. Clinical Microbiology and Infection. 2020.

18. Quigley AL, Stone H, Nguyen PY, Chughtai AA, Maclntyre CR. Estimating the burden of COVID-19 on the Australian healthcare workers and health system during the first six months of the pandemic. International journal of nursing studies. 2021;114:103811.

19. Laursen J, Petersen J, Didriksen M, Iversen K, Ullum H. Prevalence of SARS-CoV-2 IgG/IgM antibodies among Danish and Swedish Falck emergency and non-emergency healthcare workers. International Journal of Environmental Research and Public Health. 2021;18(3):923.

20. Rostami A, Sepidarkish M, Leeflang M, Riahi SM, Shiadeh MN, Esfandyari S, et al. SARS-CoV-2 seroprevalence worldwide: a systematic review and meta-analysis. Clinical Microbiology and Infection. 2020.

21. Poustchi H, Darvishian M, Mohammadi Z, Shayanrad A, Delavari A, Bahadorimonfared A, et al. SARSCoV-2 antibody seroprevalence in the general population and high-risk occupational groups across 18 cities in Iran: a population-based cross-sectional study. The Lancet Infectious Diseases. 2021;21(4):473-81.

22. Rudberg A-S, Havervall S, Månberg A, Falk AJ, Aguilera K, Ng H, et al. SARS-CoV-2 exposure, symptoms and seroprevalence in healthcare workers in Sweden. Nature communications. 2020;11(1):1-8.

23. Galanis P, Vraka I, Fragkou D, Bilali A, Kaitelidou D. Seroprevalence of SARS-CoV-2 antibodies and associated factors in health care workers: a systematic review and meta-analysis. Journal of Hospital Infection. 2020.

24. Mitchell R, Ogunremi T, Astrakianakis G, Bryce E, Gervais R, Gravel D, et al. Impact of the 2009 influenza A (H1N1) pandemic on Canadian health care workers: a survey on vaccination, illness, absenteeism, and personal protective equipment. American journal of infection control. 2012;40(7):611-6.

25. Iversen K, Bundgaard H, Hasselbalch RB, Kristensen JH, Nielsen PB, Pries-Heje M, et al. Risk of COVID-19 in health-care workers in Denmark: an observational cohort study. The Lancet Infectious Diseases. 2020;20(12):1401-8.

26. Hansen $\mathrm{CH}$, Michlmayr D, Gubbels SM, Mølbak K, Ethelberg S. Assessment of protection against reinfection with SARS-CoV-2 among 4 million PCR-tested individuals in Denmark in 2020: a population-level observational study. The Lancet. 2021;397(10280):1204-12.

27. Erfani A, Shahriarirad R, Ranjbar K, Mirahmadizadeh A, Moghadami M. Knowledge, attitude and practice toward the novel coronavirus (COVID-19) outbreak: a population-based survey in Iran. Bull World Health Organ. 2020;30(10.2471). 
28. Harvey RA, Rassen JA, Kabelac CA, Turenne W, Leonard S, Klesh R, et al. Association of SARS-CoV-2 seropositive antibody test with risk of future infection. JAMA internal medicine. 2021;181(5):672-9.

29. Abu-Raddad LJ, Chemaitelly H, Coyle P, Malek JA, Ahmed AA, Mohamoud YA, et al. SARS-CoV-2 reinfection in a cohort of 43,000 antibody-positive individuals followed for up to 35 weeks. medRxiv. 2021.

30. Larson D, Brodniak SL, Voegtly LJ, Cer RZ, Glang LA, Malagon FJ, et al. A Case of Early Re-infection with SARS-CoV-2. Clinical infectious diseases: an official publication of the Infectious Diseases Society of America. 2020.

31. Iwasaki A. What reinfections mean for COVID-19. The Lancet Infectious Diseases. 2021;21(1):3-5.

\section{Figures}

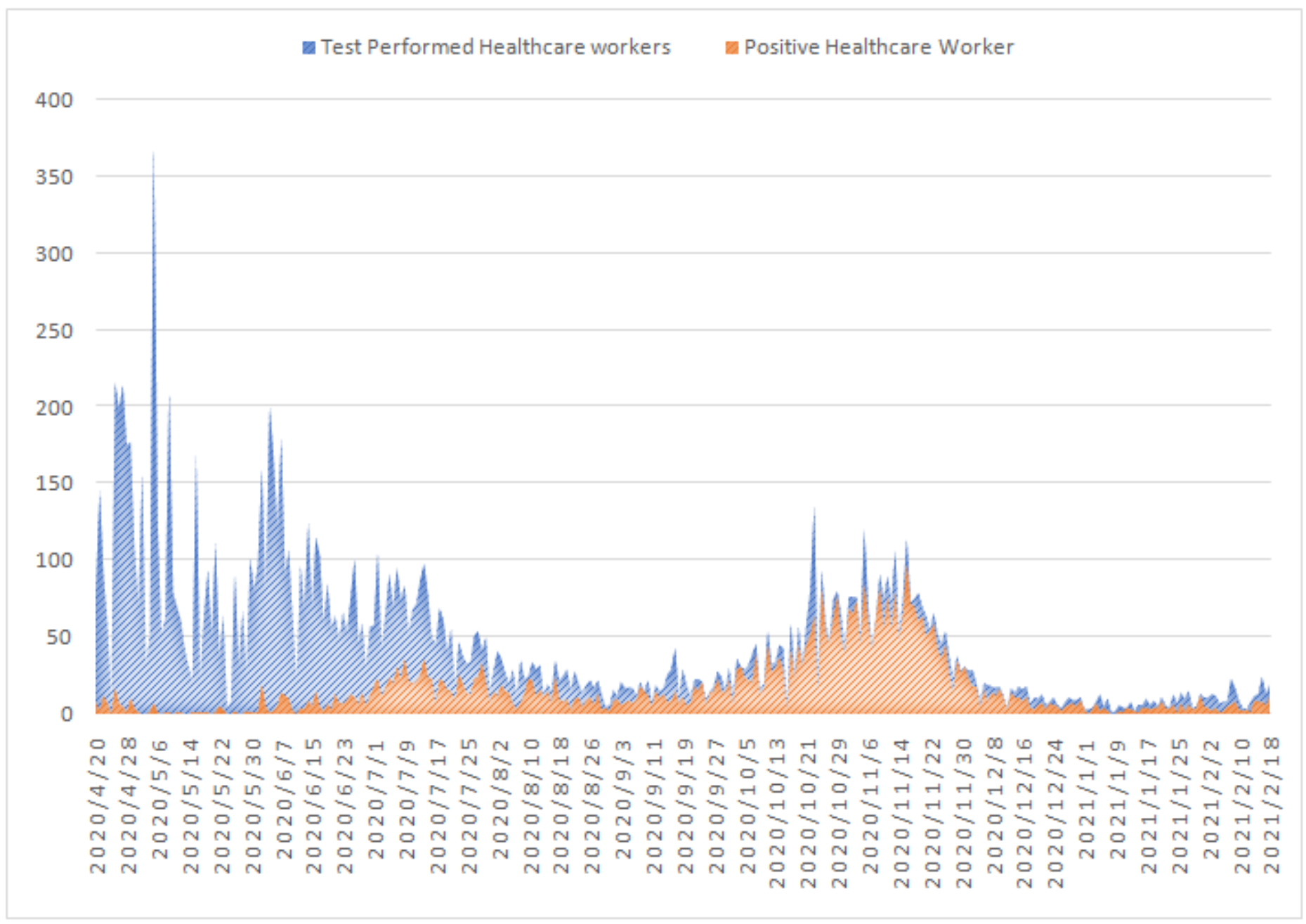

\section{Figure 1}

Positive COVID-19 among healthcare workers compared to total test performed among healthcare workers. 


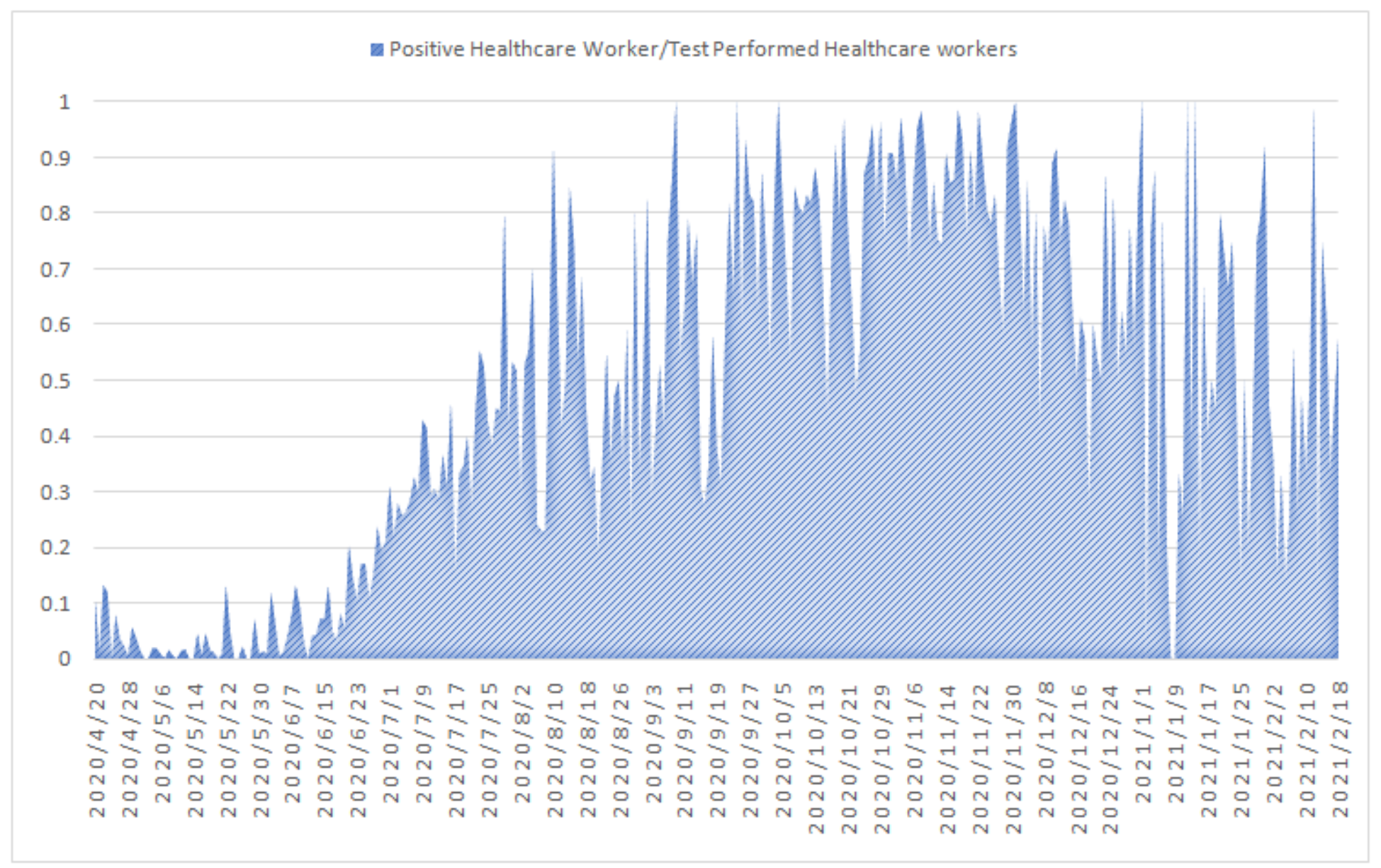

Figure 2

Proportion of positive COVID-19 among healthcare workers compared to total test performed among healthcare workers. 


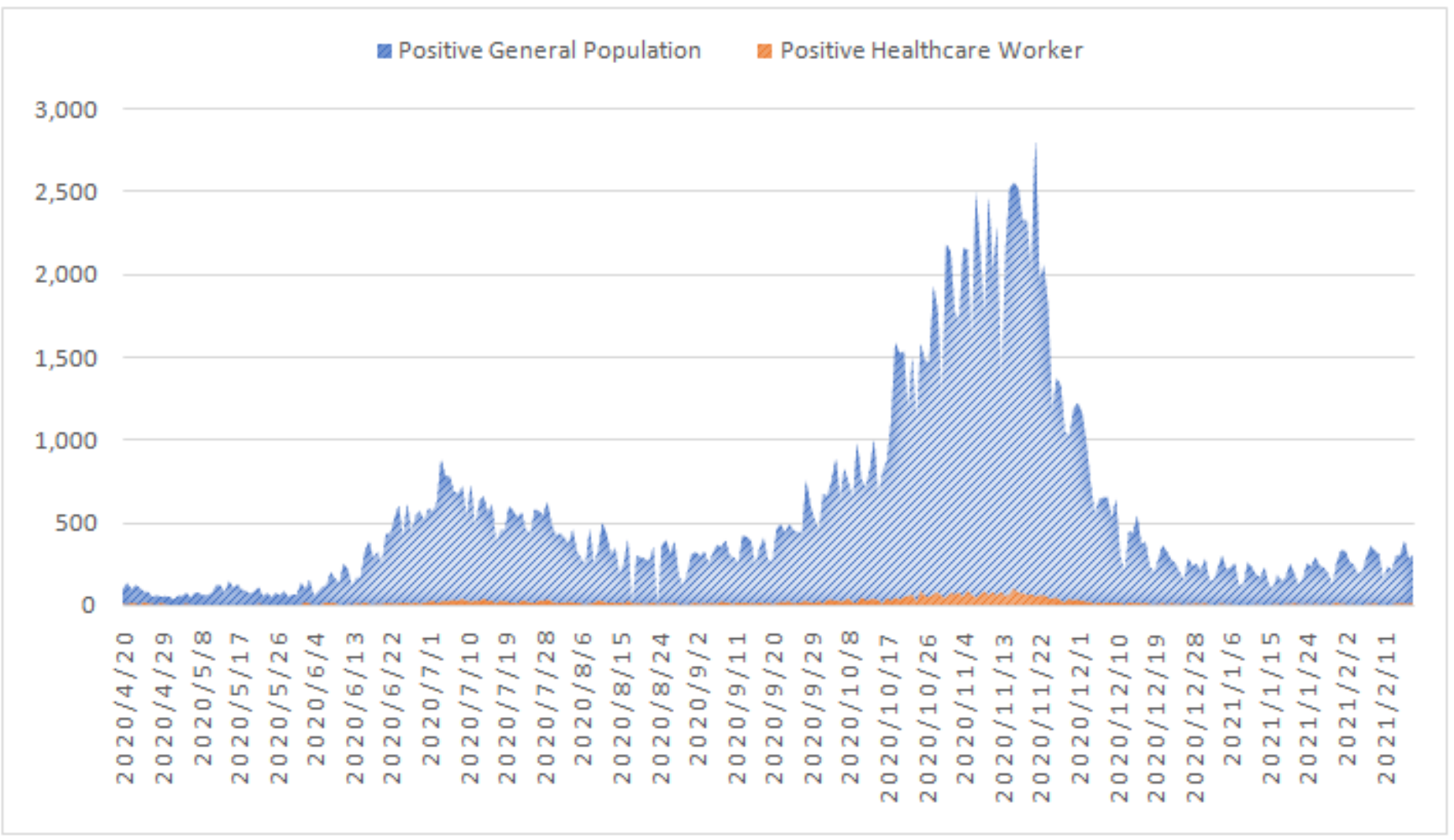

\section{Figure 3}

Frequency of positive COVID-19 among the general population and healthcare workers. 
0.25

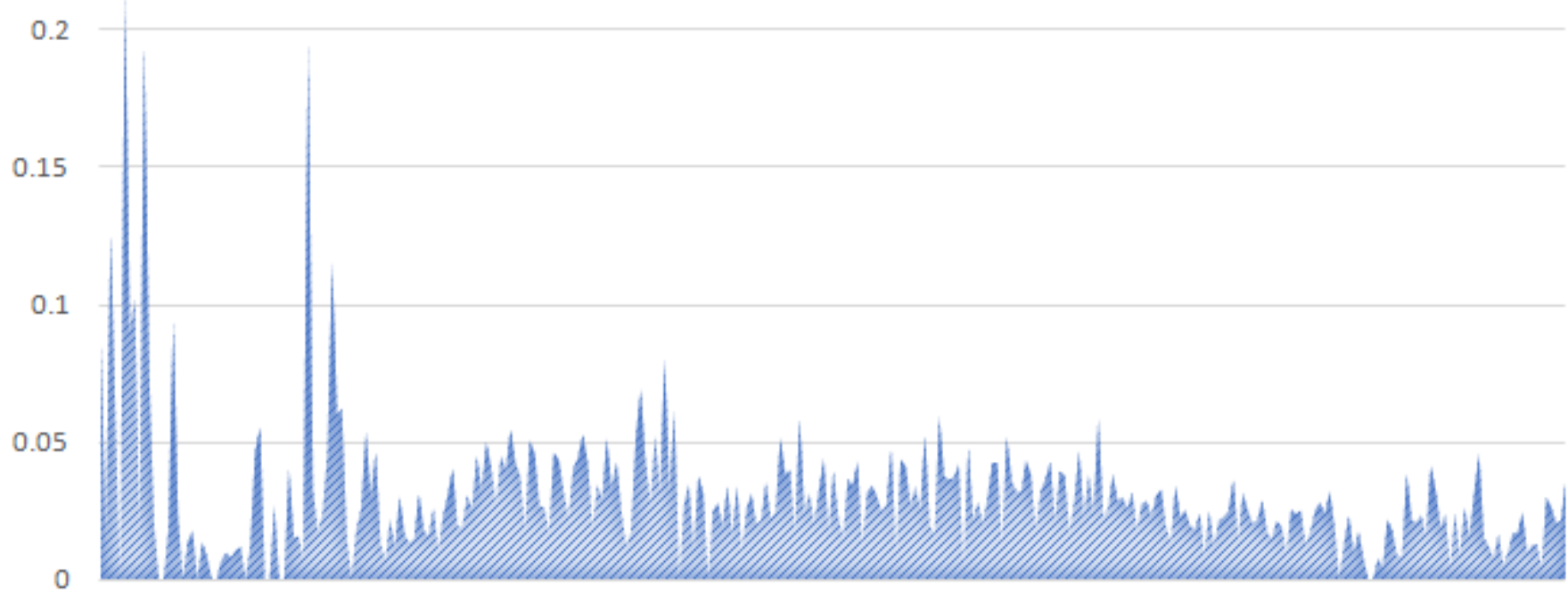

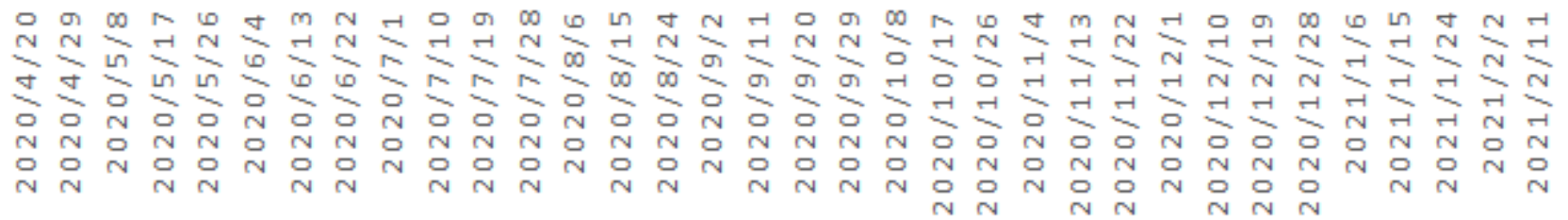

Figure 4

Proportion of positive COVID-19 among healthcare workers compared to total positive cases of COVID-19 in the general population 


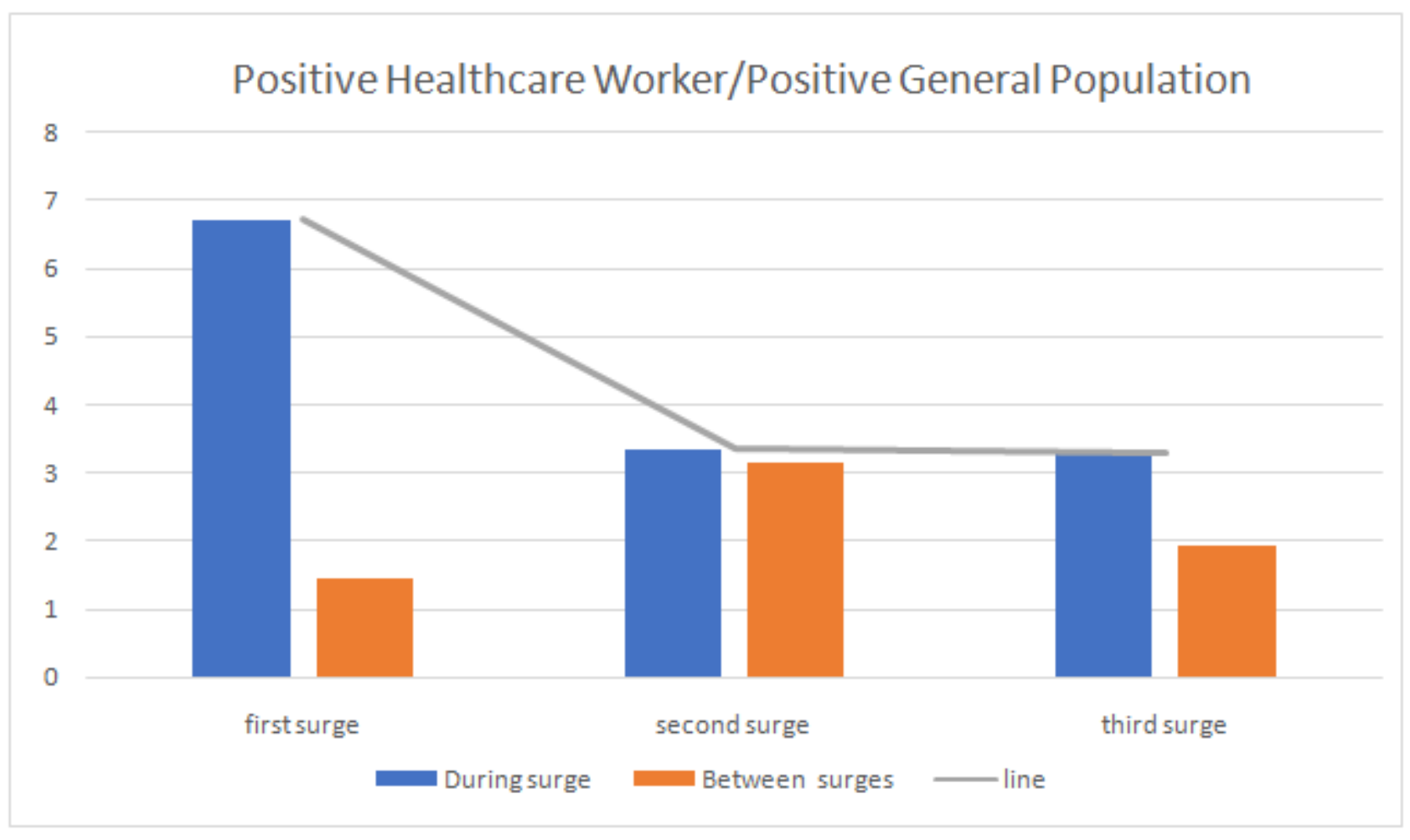

Figure 5

Comparison of the proportion of COVID-19 among HCWs to the general population based on the three COVID-19 surges in Fars province, Southern Iran. (First surge: 1th of June, 2020 - 15th August 2020, Second surge: 28th September 2020 - 12th December 2020, Third surge: 21th March 2021 - 18th February 2021) 